also positively correlated with the presence of knee beam hardening artifact. Compared to standard setting, the use of R2 settings decreased sensitivity $(0.79$ [95Cl: $0.65 ; 0.88]$ versus 0.90 [95Cl: $0.78 ; 0.96]$ and increased specificity $(0.86$ [95Cl: $0.71 ; 0.93)$ versus 0.63 [95Cl: $0.47 ; 0.77]$ ( $p<0.001)$.

Conclusion: Applying a ratio of 1.28 and a minimum attenuation of $170 \mathrm{HU}$ (R2 settings) in DECT post-processing eliminates the majority of the artifacts located on the lower limbs, particularly the clumpy artifact and the beam hardening artifact.

REFERENCES :

[1] Neogi T, Jansen TLTA, Dalbeth N, Fransen J, Schumacher HR, Berendsen D, et al. 2015 Gout classification criteria: an American College of Rheumatology/European League Against Rheumatism collaborative initiative. Ann Rheum Dis. 2015;74:1789-98.

[2] Mallinson PI, Coupal T, Reisinger C, Chou H, Munk PL, Nicolaou S, et al. Artifacts in dual-energy CT gout protocol: a review of 50 suspected cases with an artifact identification guide. AJR 2014;203:W103-109.

Disclosure of Interests: None declared.

DOI: 10.1136/annrheumdis-2021-eular.1275

\section{POS1130 PHARMACOKINETICS AND SERUM URATE LOWERING EFFECTS OF AR882, A NOVEL, POTENT AND SELECTIVE URICOSURIC AGENT, IN PATIENTS WITH GOUT}

Z. Shen ${ }^{1}$, E. Polvent ${ }^{1}$, V. Hingorani ${ }^{1}$, A. Clouser Roche ${ }^{1}$, C. Colton ${ }^{1}$, R. Yan ${ }^{1}$, S. Yan ${ }^{1}$, L. T. Yeh ${ }^{1} .{ }^{1}$ Arthrosi Therapeutics Inc, Laguna Hills, California, United States of America

Background: AR882 is a novel, potent and selective uric acid transporter 1 (URAT1) inhibitor in Phase 2 development for the treatment of hyperuricemia and gout. In Phase 1 healthy subject studies, AR882 demonstrated good dose proportionality, a long effective half-life and sustained serum urate (sUA) lowering effects, consequently making it suitable for once-daily dosing. In addition, AR882 was well tolerated following single and multiple dosing.

Objectives: This Phase 2a study evaluated the pharmacokinetics (PK), pharmacodynamics (PD), and safety of AR882 following monotherapy in patients with gout. PK and sUA lowering effects in patients with renal impairment and normal renal function were also compared.

Methods: A total of 30 adults with gout (sUA $>7 \mathrm{mg} / \mathrm{dL}$ ) were enrolled and 28 had post-baseline PKPD assessments performed. AR882 was administered at escalating doses of 25,50 and $75 \mathrm{mg}$, with each dose administered once daily for 7 days. Serial blood samples were collected to measure AR882 PK and sUA levels at the end of each treatment week. Urine samples were collected for assessment of uric acid excretion. Laboratory safety tests, vital signs, and electrocardiograms were collected throughout the study.

Results: Following once daily administration in patients with gout, AR882 exposures increased dose proportionally between 25 and $75 \mathrm{mg}$. Mean sUA levels were maximally reduced from baseline (mean $8.9 \mathrm{mg} / \mathrm{dL}$ ) to $5.6,4.2$ and $3.2 \mathrm{mg} / \mathrm{dL}$ at the $25-, 50-$ and $75-\mathrm{mg}$ dose levels, respectively. The corresponding percent reductions were $36.8 \%, 52.7 \%$ and $61.5 \%$. The effects were sustained throughout the entire dosing day with minimal fluctuation. In patients receiving $25 \mathrm{mg}$ AR882 ( $\mathrm{N}=9), 56 \%$ achieved sUA levels below $6 \mathrm{mg} /$ dL. At $50 \mathrm{mg}(\mathrm{N}=28), 96 \%$ of patients had sUA levels below $6 \mathrm{mg} / \mathrm{dL}$ and $93 \%$ were below $5 \mathrm{mg} / \mathrm{dL}$. At the $75 \mathrm{mg}$ dose $(\mathrm{N}=8)$, all patients $(100 \%)$ achieved levels below $5 \mathrm{mg} / \mathrm{dL}$, and $88 \%$ were below $4 \mathrm{mg} / \mathrm{dL}$. Among these 28 gout patients, 17 patients had normal renal function (CrCL $>90 \mathrm{~mL} / \mathrm{min}$ ) and 11 patients had mild impairment ( $\mathrm{CrCL} 60-90 \mathrm{~mL} / \mathrm{min})$. AR882 exposures were similar between patients with mild renal impairment and those with normal renal function. Both groups showed nearly identical sUA lowering effects following each 7-day dosing period. Response rates were also similar between these groups, including at the 50 and $75 \mathrm{mg}$ dose levels. Consistent with its sUA lowering effects, dose-dependent increases in fractional excretion of uric acid were observed across all doses.

AR882 was well tolerated at all doses tested. All AEs were mild or moderate in severity and most were considered not related to study treatment. There were no serious $\mathrm{AES}$ or $\mathrm{AEs}$ resulting in study drug discontinuation. There were no clinically significant laboratory or ECG abnormalities noted.

Conclusion: In patients with gout, AR882 exhibited dose proportional increases in plasma exposure and dose-dependent reductions in SUA. Nearly all patients had sUA levels below $5 \mathrm{mg} / \mathrm{dL}$ at doses $\geq 50 \mathrm{mg}$, and about $90 \%$ of patients had sUA levels $<4 \mathrm{mg} / \mathrm{dL}$ at $75 \mathrm{mg}$ dosing. Similar PK exposures and SUA lowering effects were observed in patients with mild renal impairment compared to those with normal renal function. Current data suggest that AR882 $50 \mathrm{mg}$ may offer improved efficacy over existing standard-of-care for gout and AR882 $75 \mathrm{mg}$ may have utility in the treatment of patients with severe or refractory gout disease.

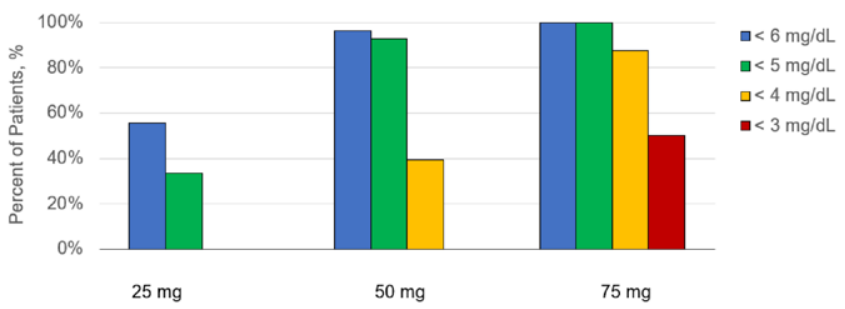

Figure 1. Percent of Patients with sUA below $6 \mathrm{mg} / \mathrm{dL}, 5 \mathrm{mg} / \mathrm{dL}, 4 \mathrm{mg} / \mathrm{dL}$ or $3 \mathrm{mg} / \mathrm{dL}$ Following Once-Daily Oral Doses of AR882.

Table 1. Mean (SD) sUA Levels Following Once-Daily Oral Doses of AR882.

\begin{tabular}{lccccc}
\hline \multirow{2}{*}{ Treatment } & \multirow{2}{*}{$\mathrm{N}$} & \multicolumn{4}{c}{ Time (hr), sUA (mg/dL) } \\
\cline { 3 - 6 } & & 0 & 6 & 12 & 24 \\
\hline Baseline & 30 & $8.8(1.1)$ & $8.8(1.1)$ & $9.0(1.0)$ & $8.9(1.1)$ \\
$25 \mathrm{mg}$ & 9 & $6.1(0.4)$ & $5.6(0.4)$ & $5.6(0.3)$ & $5.8(0.4)$ \\
$50 \mathrm{mg}$ & 28 & $4.7(0.9)$ & $4.2(0.9)$ & $4.3(0.8)$ & $4.7(1.0)$ \\
$75 \mathrm{mg}$ & 8 & $3.7(1.1)$ & $3.2(0.9)$ & $3.2(0.8)$ & $3.6(1.0)$ \\
$50 \mathrm{mg}$ (normal renal function) & 17 & $4.8(1.1)$ & $4.3(1.1)$ & $4.4(0.9)$ & $4.8(1.2)$ \\
$50 \mathrm{mg}$ (mild impairment) & 11 & $4.5(0.6)$ & $4.1(0.6)$ & $4.3(0.5)$ & $4.6(0.6)$
\end{tabular}

Disclosure of Interests: Zancong Shen Employee of: Arthrosi Therapeutics Inc, Elizabeth Polvent Employee of: Arthrosi Therapeutics Inc, Vijay Hingorani Consultant of: Arthrosi Therapeutics Inc, Andrea Clouser Roche Employee of: Arthrosi Therapeutics Inc, Chris Colton Employee of: Arthrosi Therapeutics Inc, Rongzi Yan Employee of: Arthrosi Therapeutics Inc, Shunqi Yan Employee of: Arthrosi Therapeutics Inc, Li-Tain Yeh Employee of: Arthrosi Therapeutics Inc. DOI: 10.1136/annrheumdis-2021-eular.1284

\section{\begin{tabular}{|l|l}
\hline POS1131 THE INCIDENCE AND PREVALENCE OF \\
\hline
\end{tabular} CARDIOVASCULAR DISEASES IN GOUT: A SYSTEMATIC REVIEW AND META-ANALYSIS}

P. Cox ${ }^{1}$, S. Gupta ${ }^{2}$, S. S. Zhao ${ }^{3}$, D. Hughes ${ }^{4} .{ }^{1}$ University of Liverpool, Institute of Translational Medicine, Liverpool, United Kingdom $;{ }^{2}$ University of Liverpool, School of Medicine, Liverpool, United Kingdom; ${ }^{3}$ University of Liverpool, Institute of Ageing and Chronic Disease, Liverpool, United Kingdom; ${ }^{4}$ University of Liverpool, Department of Health Data Science, Liverpool, United Kingdom

Background: Gout is an inflammatory crystal arthropathy characterised by hyperuricaemia and sodium urate crystal deposition. Both gout and subclinical hyperuricaemia are associated with adverse cardiovascular outcomes. Several studies have found gout to cause an increased risk of cardiovascular disease, but the evidence is not unanimous. The conflicting evidence has made it difficult to establish the extent of the cardiovascular risk to patients with gout.

Objectives: To describe the incidence and prevalence of cardiovascular disease in gout, compare these results with non-gout controls.

Methods: PubMed, Scopus and Web of Science were systematically searched in January 2021 for studies reporting prevalence of any cardiovascular disease in a gout population. Studies with non-representative sampling, where a cohort had been used in another study, small sample size $(<100)$ and where gout could not be distinguished from other rheumatic conditions were excluded. Sample size, prevalence of the investigated cardiovascular disease, definition of gout and cardiovascular disease, demographic data, data source and any comparisons with non-gout controls were extracted from each study. Where prevalence data was reported in $\geq 3$ cohorts meta-analysis was performed using random-effect models.

Results: Of the 6164 titles identified, 105 full texts were assessed for eligibility with 30 included in the review, producing a gout population of $1,125,988$. Pooled prevalence estimates were calculated for six cardiovascular diseases: heart failure $(8.73 \%$; 95\% confidence interval $(\mathrm{Cl}), 2.85-23.76)$, cerebrovascular accident $(4.27 \%$; $95 \% \mathrm{Cl}, 1.83-9.67)$, myocardial infarction $(2.82 \% ; 95 \% \mathrm{Cl}, 1.58$ $-5.01)$, venous thromboembolism $(2.05 \%$; $95 \% \mathrm{Cl}, 1.22-3.43)$, hypertension $(63.94 \% ; 95 \% \mathrm{Cl}, 24.51-90.64)$ and cardiovascular mortality $(4.75 \% ; 95 \% \mathrm{Cl}$ $3.56-6.31$ ). Twenty studies reported comparisons with non-gout controls, illustrating an increased risk in the gout group across all cardiovascular diseases, particularly for myocardial infarction.

Conclusion: Cardiovascular diseases are more prevalent in patients with gou and should prompt vigilance from clinicians to the need to assess and stratify cardiovascular risk. These results are in line with other studies which have shown an increased cardiovascular risk for sufferers of hyperuricaemia, highlighting the need for future research to explain this finding. There are limited studies in the 
literature investigating less common cardiovascular conditions, illustrating the need for future work if a more thorough picture of prevalence is to be established.

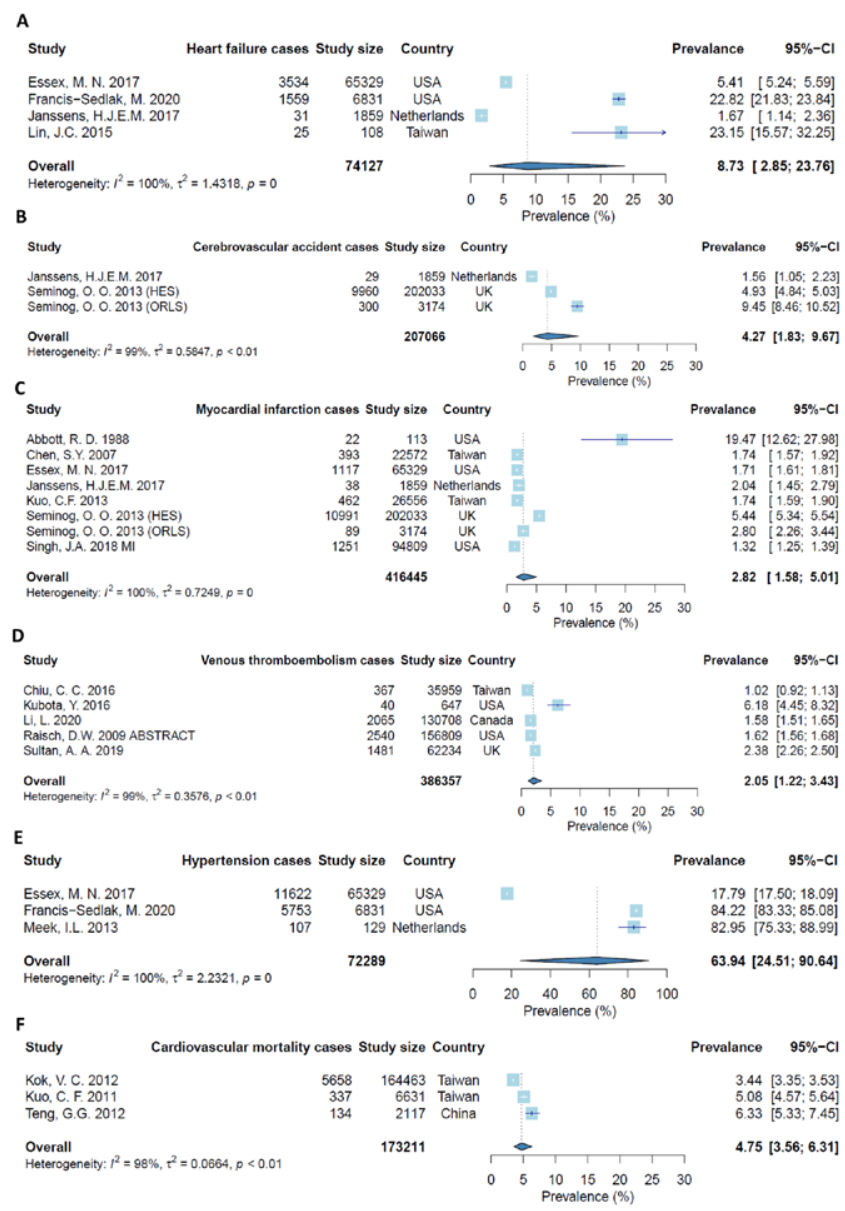

Figure 1. Forest plots of pooled prevalence of: (A) $8.73 \%$ for heart failure, (B) $4.27 \%$ for cerebrovascular accident, (C) $2.82 \%$ for myocardial infarction, (D) $2.05 \%$ for venous thromboembolism, (E) $63.94 \%$ for hypertension and (F) $4.75 \%$ for cardiovascular mortality.

Disclosure of Interests: None declared.

DOI: 10.1136/annrheumdis-2021-eular.1425

\section{POS1132 DIAGNOSTIC ACCURACY OF CONVENTIONAL RADIOGRAPHY OFTHE KNEE FOR CALCIUM PYROPHOSPHATE DEPOSITION DISEASE: AN ANCILLARY STUDY OF THE OMERACT ULTRASOUND - CPPD GROUP}

S. Sirotti ${ }^{1}$, F. Becce ${ }^{2}$, L. M. Sconfienza ${ }^{3}$, C. Pineda ${ }^{4}$, M. Gutierrez ${ }^{4}$, T. Serban ${ }^{5}$, D. Maccarter ${ }^{6}$, A. Adinolfi ${ }^{7}$, E. Naredo ${ }^{8}$, A. Scanu ${ }^{9}$, C. A. Scirè ${ }^{10}$, I. Möller ${ }^{11}$, P. Sarzi-Puttini ${ }^{1}$, A. Abhishek ${ }^{12}$, H. Choi ${ }^{13}$, N. Dalbeth ${ }^{14}$, S. Tedeschi ${ }^{15}$, M. A. D'agostino ${ }^{16}, \mathrm{H}$. Keen ${ }^{17}$, L. Terslev ${ }^{18}$, A. lagnocco ${ }^{19}$, G. Filippou on behalf of OMERACT Ultrasound working group, subgroup CPPD. ${ }^{1}$ Sacco Hospital, Rheumatology Department, Milano, Italy; ${ }^{2}$ Lausanne University Hospital, Department of Diagnostic and Interventional Radiology, Lausanne, Switzerland; ${ }^{3}$ University of Milan, Department of Biomedical Sciences for Health, Milano, Italy; ${ }^{4}$ National Rehabilitation Institute, Division of Musculoskeletal and Rheumatic Diseases, Ciudad de México, Mexico; ${ }^{5}$ Hospital La Colletta, Rheumatology Department, Arenzano, Italy; ${ }^{6}$ North Valley Hospital, Rheumatology Department, Whitefish, United States of America; ${ }^{7}$ Niguarda, Rheumatology Unit, Milan, Italy; ${ }^{8}$ Hospital Universitario Fundación Jiménez Díaz, Department of Rheumatology and Joint and Bone Research Unit, Madrid, Spain: ${ }^{9}$ University of Padua, Department of Medicine-DIMED, Rheumatology Unit, Padova, Italy: ${ }^{10}$ Società Italiana di Reumatologia, Epidemiology Research Unit, Milan, Italy; ${ }^{11}$ Instituto Poal de Reumatologia, Rheumatology, Barcelona, Spain; ${ }^{2}$ University of Nottingham, Academic Rheumatology, Nottingham, United
Kingdom; ${ }^{13}$ Massachusetts General Hospital Department of Medicine, Division of Rheumatology, Allergy, and Immunology, Boston, United States of America;

${ }^{14}$ The University of Auckland, Bone and Joint Research Group, Department of Medicine, Auckland, New Zealand; ${ }^{15}$ Brigham And Women's Hospital, Division of Rheumatology, Inflammation and Immunity, Boston, United States of America; ${ }^{16}$ Agostino Gemelli University Policlinic, Rheumatology Department, Roma, Italy: ${ }^{17}$ The University of Western Australia, School of Medicine, Murdoch, Perth, Australia: ${ }^{18}$ Rigshospitalet, Center for Rheumatology and Spine Diseases, København, Denmark; ${ }^{19}$ University of Turin, Academic Rheumatology Centre, Department of Clinical and Biological Sciences, Torino, Italy

Background: Conventional Radiography (CR) has been widely used in the assessment of knee chondrocalcinosis (CC) and is still considered one of the most important diagnostic methods for the diagnosis. However, there are very few studies that examine the diagnostic accuracy of CR compared to histology of the knee tissues.

Objectives: To assess the diagnostic accuracy of $\mathrm{CR}$ of the knee in Calcium Pyrophosphate Deposition Disease (CPPD) by using the recently created definitions for CPPD in CR of the ACR/EULAR taskforce for the new classification criteria for CPPD.

Methods: This is an ancillary study of the Criterion Validity of Ultrasound in CPPD study [1]. Consecutive patients with osteoarthritis $(\mathrm{OA})$ awaiting total knee replacement were enrolled in 4 centres from Romania, Italy, USA and Mexico. All patients underwent $\mathrm{CR}$ of the knees taken maximum 6 months before surgery, in posterior-anterior weight baring and lateral projections. DICOM files of the radiographs were anonymised and read independently by two musculoskeletal radiologists with experience in microcrystalline arthropathies. For each patient, a dichotomic score was used (absence/presence of $\mathrm{CC}$ ) at the level of the menisci and tibiofemoral hyaline cartilage by each reader. The definitions of the ACR/EULAR taskforce for identification of CPPD in CR were used in this study [paper in preparation]. According to these definitions CPPD in CR appears as "linear or punctate opacities in the region of fibro- or hyaline articular cartilage/synovial membrane or joint capsule/ within tendons or entheses that are distinct from denser, nummular radio-opaque deposits due to basic calcium phosphate deposition." In case of disagreement a consensus decision was taken by both radiologists after discussion of the case. Menisci and the hyaline cartilage were analysed using compensated polarized ligh microscopy as described previously [1], patients were considered positive for CPPD if at least one of their tissue specimens revealed the presence of calcium pyrophosphate crystals. All examiners were blind to each other's findings.

Results: We enrolled 33 patients with OA (61\% female, mean age $69 y$ y). The accuracy values of $C R$ in the various sites of the knee are indicated in Table 1. CR demonstrated to be a specific exam for identification of CPPD at the knee, but sensitivity remains low in all sites and in the overall evaluation. Identification of CPPD appears challenging and this could be due to the advanced grade of $\mathrm{OA}$ in our cohort of patients. Advanced degeneration, dislocation of the menisc and thinning of the hyaline cartilage in these patients is frequent and the eventual presence of calcific deposits in one of these structures could overlap with other anatomical structures making the exact localisation difficult. According to the results of the predictive values, the presence of typical deposition on $\mathrm{CR}$ allows a definite confirmation of the diagnosis, but a negative radiography does not exclude CPPD as testified by the low negative predictive value.

Table 1. Sensitivity, specificity, PPV, NPV, accuracy and AUC of CR for identification of CPPD by using the new ACR/EULAR taskforce definitions.

\begin{tabular}{lcccc}
\hline & Medial meniscus & Lateral meniscus & Hyaline cartilage & Overall \\
\hline Sensitivity & $22 \%$ & $33 \%$ & $31 \%$ & $42 \%$ \\
Specificity & $100 \%$ & $100 \%$ & $85 \%$ & $90 \%$ \\
Positive predictive value & $100 \%$ & $100 \%$ & $67 \%$ & $80 \%$ \\
Negative predictive value & $56 \%$ & $60 \%$ & $55 \%$ & $61 \%$ \\
Accuracy & $61 \%$ & $68 \%$ & $58 \%$ & $66 \%$ \\
AUC & 0.6 & 0.7 & 0.6 & 0.7
\end{tabular}

Conclusion: CR has been extensively used for the diagnosis of OA and CPPD and has been tested previously for diagnostic accuracy. The results of our study confirm that the presence of typical CPPD calcifications, as defined by the ACR EULAR task force, are highly specific but have low sensitivity for disease identification when using CR. Absence of CPPD on CR does not exclude the diagnosis. REFERENCES:

[1] Filippou G, et al. Criterion validity of ultrasound in the identification of calcium pyrophosphate crystal deposits at the knee: an OMERACT ultrasound study. Ann Rheum Dis 2020. doi:10.1136/annrheumdis-2020-217998

Disclosure of Interests: None declared.

DOI: 10.1136/annrheumdis-2021-eular.1437 